\title{
Actions and symmetries of NSR superstrings and D-strings
}

\author{
Friedemann Brandt ${ }^{a, b}$, Alexander Kling ${ }^{c}$, Maximilian Kreuzer $^{c}$ \\ ${ }^{a}$ Max-Planck-Institute for Mathematics in the Sciences, Inselstr. 22-26, D-04103 Leipzig, Germany \\ ${ }^{b}$ Institut für Theoretische Physik, Universität Hannover, Appelstr. 2, D-30167 Hannover, Germany \\ ${ }^{c}$ Institut für Theoretische Physik, Technische Universität Wien, \\ Wiedner Hauptstraße 8-10, A-1040 Vienna, Austria
}

\begin{abstract}
We present all NSR superstring and super-D-string actions invariant under a set of prescribed gauge transformations, and characterize completely their global symmetries. In particular we obtain locally supersymmetric Born-Infeld actions on general backgrounds in a formulation with extra target space dimensions. The nontrivial global symmetries of the superstring actions correspond to isometries of the background, whereas super-D-string actions can have additional symmetries acting nontrivially also on the coordinates of the extra dimensions.
\end{abstract}

PACS numbers: 11.25.-w, 11.30.-j

This letter is devoted to locally supersymmetric worldsheet actions for superstrings and super-D-strings and to their symmetries. For the standard spinning string in a flat background [1], such an action has been known for more than 20 years [2]. It played an important rôle in string theory, in particular as starting point for covariant quantization, and was extended to general backgrounds [3]. In this letter we present analogous actions for the super-D-string, i.e. locally supersymmetric world-sheet actions of the Born-Infeld type. To our knowledge, such actions have not been explicitly constructed previously, except for the D2-brane $\llbracket$. In addition we characterize completely the global symmetries of these actions.

As it is well known the tension of a super-p-brane may be generated dynamically as the flux of a world-volume p-form gauge field [5]. Since D-branes are described by Born-Infeld type actions it is natural to construct a manifestly S-dual IIB superstring by assembling the BornInfeld field and a tension generating gauge field into an $S L(2)$ doublet [6]. The resulting theory is in a sense twelve-dimensional. This is quite apparent in our formulation which uses actually a twelve-dimensional target space. The two "extra dimensions" get frozen: the values of their coordinates are integration constants by the equations of motion. In the light of this interpretation in terms of extra dimensions, it is quite interesting that the symmetries of the super-D-string action are not exhausted by the standard isometries of the "ordinary" (ten-dimensional) target space: rather, there can be additional symmetries which act nontrivially also on the coordinates of the extra dimensions. Our results may thus be of interest also in view of a possible twelve-dimensional theory underlying IIB superstring theory [7]. Inspired by such considerations (and for the sake of generality) we allow for an arbitrary number of gauge fields and corresponding "extra dimensions", thereby including Dstrings but not restricting to them.

a. Field content and gauge symmetries. The results reported here were obtained by a BRST-cohomological analysis whose details will be presented elsewhere. The input of this analysis is the field content and a set of gauge transformations of the fields. The fields are those of the $2 d$ supergravity multiplet $\left\{e_{m}^{a}, \chi_{m}^{\alpha}, S\right\} \quad(m, a, \alpha$ are $2 d$ world-sheet, Lorentz and spinor indices, respectively), an arbitrary number of "matter multiplets" $\left\{X^{M}, \psi_{\alpha}^{M}, F^{M}\right\}$ and an arbitrary number of gauge multiplets $\left\{A_{m}^{i}, \lambda_{\alpha}^{i}, \phi^{i}\right\} . \quad e_{m}^{a}$ and $\chi_{m}^{\alpha}$ are the world-sheet zweibein and gravitino, respectively, the $A_{m}^{i}$ are abelian gauge fields, and the $X^{M}$ may be regarded as coordinates of an enlarged target space. All fields, including the auxiliary fields are real (we use a Majorana-Weyl basis for the $\gamma$-matrices and the signature of the world-sheet metric is $\eta_{a b}=\operatorname{diag}(1,-1)$; furthermore $\varepsilon^{01}=\varepsilon_{10}=1$ and $\gamma_{*}$ is defined through $\gamma^{a} \gamma^{b}=\eta^{a b} \mathbb{1}+\varepsilon^{a b} \gamma_{*}$; our formulae apply with appropriate redefinitions also to Euclidean signature). We impose the following gauge symmetries: world-sheet diffeomorphisms, local $(1,1)$ world-sheet supersymmetry, local $2 d$ Lorentz invariance, invariance under abelian gauge transformations of the $A_{m}^{i}$, Weyl and super-Weyl invariance, and invariance under arbitrary local shifts of the field $S$. The corresponding gauge transformations of the fields are, written as BRST transformations,

$$
\begin{aligned}
s e_{m}^{a}= & \xi^{n} \partial_{n} e_{m}^{a}+\left(\partial_{m} \xi^{n}\right) e_{n}^{a}+C_{b}{ }^{a} e_{m}^{b}+C^{W} e_{m}^{a} \\
& -2 \mathrm{i} \xi^{\alpha} \chi_{m}^{\beta}\left(\gamma^{a} C\right)_{\alpha \beta} \\
s \chi_{m}^{\alpha}= & \xi^{n} \partial_{n} \chi_{m}^{\alpha}+\left(\partial_{m} \xi^{n}\right) \chi_{n}^{\alpha}-\frac{1}{4} C^{a b} \chi_{m}^{\beta} \varepsilon_{a b}\left(\gamma_{*}\right)_{\beta}^{\alpha} \\
& +\frac{1}{2} C^{W} \chi_{m}^{\alpha}+\mathrm{i} \eta^{\beta}\left(\gamma_{m}\right)_{\beta}^{\alpha} \\
& +\partial_{m} \xi^{\alpha}+\frac{1}{4} \xi^{\beta}\left(\omega_{m}^{a b} \varepsilon_{a b}\left(\gamma_{*}\right)_{\beta}^{\alpha}-\left(\gamma_{m}\right)_{\beta}^{\alpha} S\right) \\
s S= & \xi^{n} \partial_{n} S-C^{W} S+W+\mathrm{i} \xi^{\gamma}\left(\gamma^{m} C\right)_{\gamma_{\alpha}} \chi_{m}^{\alpha} S \\
& -4 \xi^{\gamma}\left(\gamma_{*} C\right)_{\gamma \alpha} \varepsilon^{n m}\left(\partial_{n} \chi_{m}^{\alpha}+\frac{1}{4} \omega_{n}^{a b} \varepsilon_{a b} \chi_{m}^{\beta}\left(\gamma_{*}\right)_{\beta}^{\alpha}\right) \\
s X^{M}= & \xi^{m} \partial_{m} X^{M}+\xi^{\alpha} \psi_{\alpha}^{M} \\
s \psi_{\alpha}^{M}= & \xi^{m} \partial_{m} \psi_{\alpha}^{M}+\frac{1}{4} C^{a b} \varepsilon_{a b}\left(\gamma_{*}\right)_{\alpha}^{\beta} \psi_{\beta}^{M}-\frac{1}{2} C^{W} \psi_{\alpha}^{M} \\
& +\xi^{\beta} C_{\beta \alpha} F^{M}-\mathrm{i} \xi^{\beta}\left(\gamma^{m} C\right)_{\beta \alpha}\left(\partial_{m} X^{M}-\chi_{m}^{\gamma} \psi_{\gamma}^{M}\right) \\
s F^{M}= & \xi^{m} \partial_{m} F^{M}-C^{W} F^{M}+\xi^{\alpha}\left(\gamma^{m}\right)_{\alpha}^{\beta}\left\{\partial_{m} \psi_{\beta}^{M}\right. \\
& -\frac{1}{4} \omega_{m}^{a b} \varepsilon_{a b}\left(\gamma_{*}\right)_{\beta}^{\gamma} \psi_{\gamma}^{M}-\chi_{m}^{\gamma} C_{\gamma \beta} F^{M}
\end{aligned}
$$




$$
\begin{aligned}
& \left.+\mathrm{i} \chi_{m}^{\gamma}\left(\gamma^{n} C\right)_{\gamma \beta}\left(\partial_{n} X^{M}-\chi_{n}{ }^{\delta} \psi_{\delta}^{M}\right)\right\} \\
s A_{m}^{i}= & \xi^{n} \partial_{n} A_{m}^{i}+\left(\partial_{m} \xi^{n}\right) A_{n}^{i}+\partial_{m} C^{i} \\
& -2 \mathrm{i} \xi^{\alpha} \chi_{m}^{\beta}\left(\gamma_{*} C\right)_{\beta \alpha} \phi^{i}-\xi^{\alpha}\left(\gamma_{m}\right)_{\alpha}{ }^{i} \lambda_{\beta} \\
s \phi^{i}= & \xi^{n} \partial_{n} \phi^{i}-C^{W} \phi^{i}+\xi^{\alpha}\left(\gamma_{*}\right)_{\alpha}{ }^{i} \lambda_{\beta}^{i} \\
s \lambda_{\beta}^{i}= & \xi^{n} \partial_{n} \lambda_{\beta}^{i}+\frac{1}{4} C^{a b} \varepsilon_{a b}\left(\gamma_{*}\right)_{\beta}{ }^{\gamma} \lambda_{\gamma}^{i}-\frac{3}{2} C^{W} \lambda_{\beta}^{i} \\
& +\mathrm{i} \xi^{\alpha}\left\{-\left(\gamma_{*} \gamma^{m} C\right)_{\alpha \beta}\left(\partial_{m} \phi^{i}-\chi_{m} \gamma_{*} \lambda^{i}\right)\right. \\
& +\left(\gamma_{*} C\right)_{\alpha \beta} \varepsilon^{m n}\left(\partial_{m} A_{n}^{i}+\chi_{m} \gamma_{n} \lambda^{i}-\mathrm{i} \chi_{n} \gamma_{*} C \chi_{m} \phi^{i}\right) \\
& \left.+\left(\gamma_{*} C\right)_{\alpha \beta} S \phi^{i}\right\}+2 \eta_{S W}^{\alpha}\left(\gamma_{*} C\right)_{\alpha \beta} \phi^{i}
\end{aligned}
$$

where $\xi^{m}$ are the ghosts of world-sheet diffeomorphisms, $\xi^{\alpha}$ are the supersymmetry ghosts, $C^{a b}$ is the Lorentz ghost, $C^{W}$ and $\eta_{S W}^{\alpha}$ are the Weyl and super-Weyl ghosts, respectively, $C^{i}$ are the ghosts associated with the gauge fields $A_{m}^{i}$, and $W$ is the ghost corresponding to the local shifts of the auxiliary field $S$. A $C$ without any index denotes the charge conjugation matrix. These gauge transformations were obtained from an analysis of the $2 d$ supergravity algebra in presence of the matter and gauge multiplets [8] (the analysis is analogous to the superspace analysis in 90). The corresponding BRST transformations of the ghosts are such that $s^{2}=0$.

b. Gauge invariant actions. Owing to the use of auxiliary fields, the algebra of the gauge transformations closes off-shell. As a consequence, neither the BRST transformations (1), nor the BRST transformations of the ghosts contain antifields. This allows one to determine the action functionals which are invariant under the gauge transformations (11) by computing the cohomology of $s$ in the space of antifield independent local functionals with ghost number 0 . Our result of this computation is the following. The most general Lagrangian $L$ which transforms under the above gauge transformations into a total derivative and which is polynomial in the derivatives of the fields is, modulo total derivatives,

$$
\begin{aligned}
L / e= & \frac{1}{2} \partial_{m} X^{M} \partial_{n} X^{N}\left(-h^{m n} G_{M N}+\varepsilon^{m n} B_{M N}\right) \\
& +\frac{\mathrm{i}}{2} \bar{\psi}^{M} \gamma^{m} \partial_{m} \psi^{N} G_{M N}+\frac{1}{2} F^{M} F^{N} G_{M N} \\
& +\chi_{k} \gamma^{n} \gamma^{k}\left(\psi^{N} \partial_{n} X^{M}-\frac{1}{4} C \chi_{n} \bar{\psi}^{M} \psi^{N}\right) G_{M N} \\
& +\left(\frac{1}{2} F^{M} \bar{\psi}^{K} \psi^{N}-\mathrm{i} \bar{\psi}^{N} \gamma^{m} \psi^{M} \partial_{m} X^{K}\right) \Gamma_{N K M} \\
& +\frac{1}{4}\left(F^{M} \bar{\psi}^{K} \gamma_{*} \psi^{N}-\mathrm{i} \bar{\psi}^{N} \gamma^{m} \gamma_{*} \psi^{M} \partial_{m} X^{K}\right) H_{N K M} \\
& -\frac{\mathrm{i}}{12} \chi_{m} \gamma^{n} \gamma^{m} \psi^{M} \bar{\psi}^{N} \gamma_{n} \gamma_{*} \psi^{K} H_{M N K} \\
& +\frac{1}{16} \bar{\psi}^{M}\left(\mathbb{1}+\gamma_{*}\right) \psi^{N} \bar{\psi}^{K}\left(\mathbb{1}+\gamma_{*}\right) \psi^{L} R_{K M L N} \\
& +\varepsilon^{m n} D_{i} \partial_{m} A_{n}^{i}+\frac{\mathrm{i}}{4} \bar{\psi}^{M} \psi^{N} \phi^{i} \partial_{N} \partial_{M} D_{i} \\
& +\frac{1}{2}\left(\mathrm{i} \bar{\psi}^{N} \gamma_{*} \lambda^{i}-\mathrm{i} F^{N} \phi^{i}+\chi_{m} \gamma^{m} \psi^{N} \phi^{i}\right) \partial_{N} D_{i},
\end{aligned}
$$

where $e=\operatorname{det}\left(e_{m}^{a}\right)$, and $h_{m n}$ is the world-sheet metric built from $e_{m}^{a}$. $G_{M N}, B_{M N}$ and $D_{i}$ are arbitrary functions of the $X^{M}$, except that $G_{M N}$ and $B_{M N}$ are symmetric and antisymmetric in $M$ and $N$, respectively, and $\Gamma_{M N K}, H_{M N K}$ and $R_{M N K L}$ are given by

$$
\begin{aligned}
\Gamma_{M N K} & =\frac{1}{2}\left(\partial_{M} G_{N K}+\partial_{N} G_{M K}-\partial_{K} G_{M N}\right) \\
H_{M N K} & =\partial_{M} B_{N K}+\partial_{N} B_{K M}+\partial_{K} B_{M N} \\
R_{M N K L} & =\partial_{K} \partial_{[M}(G+B)_{N] L}-\partial_{L} \partial_{[M}(G+B)_{N] K}
\end{aligned}
$$

where $\partial_{M}$ denotes differentiation with respect to $X^{M}$. The redefinitions $B_{M N}(X) \rightarrow B_{M N}(X)+\partial_{[M} f_{N]}(X)$ and $D_{i}(X) \rightarrow D_{i}(X)+k_{i}$ modify the Lagrangian only by total derivatives for any functions $f_{N}(X)$ and constants $k_{i}$.

Apart from the terms containing fields of the gauge multiplets, the Lagrangian (2) agrees with the one derived in [3] when one eliminates the auxiliary fields $F^{M}$. Hence, the cohomological analysis shows that the Lagrangian derived in 3 is in fact unique in absence of gauge multiplets (modulo total derivatives, and up to the choice of $G_{M N}$ and $B_{M N}$ ). It should be noted, however, that this uniqueness is tied to the gauge transformations (11) and may get lost when one allows that the gauge transformations get consistently deformed. E.g., one would expect that the world-sheet diffeomorphisms and supersymmetry transformations can be nontrivially deformed such that the deformed action is invariant under the deformed transformations if the background has special isometries, by analogy with the purely bosonic case 10]. Furthermore, there are certainly actions invariant under nonabelian gauge transformations of the $A_{m}^{i}$ (leading to nonabelian Born-Infeld actions, among others). The general deformation problem is currently under study.

c. Simplified action. For further discussion we shall assume in the following that the functions $D_{i}$ coincide with a subset of the fields $X^{M}$. We denote this subset by $\left\{y^{i}\right\}$ and the remaining $X^{\prime}$ 's by $x^{\mu}$,

$$
\left\{X^{M}\right\}=\left\{x^{\mu}, y^{i}\right\}, \quad D_{i}=y^{i} .
$$

In fact, this assumption is a very mild one because, except at stationary points of $D_{i}(X),(3)$ can be achieved by a field redefinition $X^{M} \rightarrow \tilde{X}^{M}=X^{M}(X)$, where this "coordinate transformation" is such that each nonconstant $D_{i}(X)$ becomes one of the $\tilde{X}$ 's. Indeed, constant $D_{i}$ give only constributions to the Lagrangian which are total derivatives and can thus be neglected, at least classically; nonconstant $D_{i}$ can be assumed to be independent by a suitable choice of basis for the gauge fields and may thus be taken as $\tilde{X}$ 's, at least locally (e.g., if $D_{1}=D_{2}$, the Lagrangian depends only on the combination $A_{m}^{1}+A_{m}^{2}$ which can be introduced as a new gauge field).

It is now easy to see that the Lagrangian (2) can actually be simplified by setting the fields $\psi^{i}, F^{i}, \lambda^{i}, \phi^{i}$ to zero. Indeed, owing to (3), the classical equations of motion for $\lambda^{i}$ and $\phi^{i}$ yield $\psi^{i}=0$ and $F^{i}=0$. The latter equations are algebraic and can be used in the Lagrangian. Then the Lagrangian does not contain $\lambda^{i}$ and $\phi^{i}$ anymore and the only remnant of the gauge multiplets are the terms $e \varepsilon^{m n} y^{i} \partial_{m} A_{n}^{i}$. This reflects that the gauge multiplets carry no dynamical degrees of freedom 
since the world-sheet is 2-dimensional. Of course, the transformations (11) must be adapted in order to provide the gauge symmetries of the simplified Lagrangian: those fields that are eliminated from the action must also be eliminated from the transformations of the remaining fields using the equations of motion of the eliminated fields. This only affects the supersymmetry transformations of $y^{i}$ and $A_{m}^{i}$. The new supersymmetry transformation of $y^{i}$ is then simply zero. This is not in contradiction with the supersymmetry algebra because the equations of motion for the $A_{m}^{i}$ give $\partial_{m} y^{i}=0$ (of course, after eliminating the fields $\psi^{i}, F^{i}, \lambda^{i}, \phi^{i}$, the supersymmetry algebra holds only on-shell). The $y^{i}$ are thus constant on-shell, their values being integration constants fixed only by initial conditions. This leads to the interpretation of the $y^{i}$ as coordinates of "frozen extra dimensions" mentioned in the beginning.

d. Born-Infeld actions. Locally supersymmetric Born-Infeld actions arise from (2) for particular choices of $G_{M N}$ and $B_{M N}$, in complete analogy to the purely bosonic case 12. For instance, consider the case with only one gauge field $\left(\left\{A_{m}^{i}\right\}=\left\{A_{m}\right\},\left\{y^{i}\right\}=\{y\}\right)$ and the following particular choice of $G_{M N}$ and $B_{M N}$,

$$
\begin{aligned}
G_{\mu \nu} & =\sqrt{1+y^{2}} g_{\mu \nu}(x), \quad B_{\mu \nu}=y b_{\mu \nu}(x) \\
G_{y y} & =G_{y \mu}=B_{y \mu}=0 .
\end{aligned}
$$

$y$ can be eliminated algebraically. Eliminating also the world-sheet zweibein $e_{m}^{a}$, the Lagrangian becomes

$$
\begin{gathered}
L= \pm \sqrt{-\operatorname{det}\left(g_{m n}+\mathcal{F}_{m n}\right)}+\ldots \\
g_{m n}=g_{\mu \nu}(x) \partial_{m} x^{\mu} \partial_{n} x^{\nu} \\
\mathcal{F}_{m n}=\partial_{m} A_{n}-\partial_{n} A_{m}-b_{\mu \nu}(x) \partial_{m} x^{\mu} \partial_{n} x^{\nu}
\end{gathered}
$$

where we have assumed $\operatorname{det}\left(g_{m n}\right)<0$ and $\operatorname{det}\left(g_{m n}+\right.$ $\left.\mathcal{F}_{m n}\right)<0$, and the nonwritten terms involve fermions. The Born-Infeld Lagrangian $L= \pm\left[+\operatorname{det}\left(g_{m n}+\right.\right.$ $\left.\left.\mathcal{F}_{m n}\right)\right]^{1 / 2}+\ldots$ for Euclidean signature $\left(\operatorname{det}\left(g_{m n}\right)>0\right)$ corresponds to $G_{\mu \nu}=\left(1-y^{2}\right)^{1 / 2} g_{\mu \nu}(x)$.

e. Global symmetries. Our second result concerns the global symmetries of the action (2). These can be obtained from the BRST cohomology in the space of antifield dependent local functionals with ghost number -1 [13. We have computed this cohomology completely and present now the resulting global symmetries for the simplified form of the action described above (without the fields $\psi^{i}, F^{i}, \lambda^{i}, \phi^{i}$ and assuming (3)). The nontrivial global symmetries (a global symmetry is called trivial when it is equal to a gauge transformation on-shell) are generated by the following transformations,

$$
\begin{aligned}
\Delta e_{m}^{a} & =0, \quad \Delta \chi_{m}^{\alpha}=0 \\
\Delta X^{M} & =\mathcal{H}^{M}, \quad \mathcal{H}^{i}=K^{i}(y), \quad \mathcal{H}^{\mu}=V^{\mu}(X) \\
\Delta \psi_{\alpha}^{\mu} & =\psi_{\alpha}^{\nu} \partial_{\nu} V^{\mu}(X) \\
\Delta F^{\mu} & =F^{\nu} \partial_{\nu} V^{\mu}(X)+\frac{1}{2} \bar{\psi}^{\nu} \psi^{\lambda} \partial_{\nu} \partial_{\lambda} V^{\mu}(X)
\end{aligned}
$$

$$
\begin{aligned}
\Delta A_{m}^{i}= & b_{M}^{i}(X) \partial_{m} X^{M}+a_{M}^{i}(X) \varepsilon_{m}{ }^{n} \partial_{n} X^{M} \\
& -\delta_{j k} A_{m}^{j} \partial_{i} K^{k}(y)-\chi_{n} \gamma_{m} \gamma^{n} \gamma_{*} \psi^{\mu} a_{\mu}^{i}(X) \\
& +\frac{i}{2} \bar{\psi}^{\mu} \gamma_{m}\left\{\gamma_{*} \psi^{\nu} \partial_{[\nu} a_{\mu]}^{i}(X)-\psi^{\nu} \partial_{[\nu} b_{\mu]}^{i}(X)\right\}
\end{aligned}
$$

where $\mathcal{H}^{M}, a_{M}^{i}$ and $b_{M}^{i}$ have to solve the following generalized Killing vector equations,

$$
\begin{aligned}
\mathcal{L}_{\mathcal{H}} G_{M N} & =-2 \delta_{i(M} a_{N)}^{i}, \\
\mathcal{L}_{\mathcal{H}} B_{M N} & =-2 \partial_{[M} p_{N]}+2 \delta_{i[M} b_{N]}^{i}
\end{aligned}
$$

for some functions $p_{M}(X)\left(\mathcal{L}_{\mathcal{H}}\right.$ is the Lie derivative along $\mathcal{H}^{M}$ and $\delta_{i M}$ is the Kronecker symbol). Note that the $p_{M}$ do not occur in the $\Delta$-transformations; however, they do contribute to the corresponding Noether currents.

The equations (7) are actually the same as the equations which also determine the symmetries of bosonic string and D-string actions [11,12 with the specification (3). In the absence of gauge fields (no $A_{m}^{i}, y^{i}, K^{i}$; $\left.\left\{\mathcal{H}^{M}\right\} \equiv\left\{V^{\mu}\right\}\right)$, they read

$$
\mathcal{L}_{V} G_{\mu \nu}=0, \quad \mathcal{L}_{V} B_{\mu \nu}=-2 \partial_{[\mu} p_{\nu]}
$$

These equations had been already discussed in 14. The first equation (\$) is just the standard Killing vector equation for $G_{\mu \nu}$. Hence, the solutions of equations (8) are those Killing vector fields of $G_{\mu \nu}$ which solve the second equation (8) (for some $p_{\mu}$ ).

The situation changes when gauge fields are present. Then equations (7) read for $M, N=\mu, \nu$ :

$$
\left(\mathcal{L}_{V}+K^{i} \partial_{i}\right) G_{\mu \nu}=0,\left(\mathcal{L}_{V}+K^{i} \partial_{i}\right) B_{\mu \nu}=-2 \partial_{[\mu} p_{\nu]},
$$

where $\mathcal{L}_{V}$ is the Lie derivative along the vector field $V^{M}$ given by $V^{i}=0, V^{\mu}=V^{\mu}(X)$. The remaining equations (7) just determine the functions $a_{M}^{i}$ and $b_{M}^{i}$,

$$
\begin{gathered}
a_{\mu}^{i}=-\mathcal{L}_{\mathcal{H}} G_{\mu i}, \quad a_{i}^{j}=-\frac{1}{2} \mathcal{L}_{\mathcal{H}} G_{i j} \\
b_{\mu}^{i}=-\mathcal{L}_{\mathcal{H}} B_{\mu i}+\partial_{i} p_{\mu}, \quad b_{i}^{j}=-\frac{1}{2} \mathcal{L}_{\mathcal{H}} B_{i j} .
\end{gathered}
$$

Here we have used that $p_{i}$ and the parts of $a_{i}^{j}$ resp. $b_{i}^{j}$ which are antisymmetric resp. symmetric in $i, j$ can be set to zero without loss of generality (the corresponding contributions to $\Delta$ can be removed by subtracting trivial global symmetries from $\Delta$ ).

The global symmetries are thus completely determined by equations (9). Note that these equations reproduce (8) for $K^{i}=0$, except that now $G_{\mu \nu}$ and $B_{\mu \nu}$ depend in general not only on the $x^{\mu}$ but also on the $y^{i}$. Hence, in general $V^{\mu}$ and $p_{\mu}$ also depend on the $y^{i}$. For the discussion of equations (9), the $y^{i}$ may be viewed as parameters of $G_{\mu \nu}$ and $B_{\mu \nu}$ rather than as coordinates of extra dimensions. Solutions to equations (9) with $K^{i}=0$ can thus be regarded as solutions to equations (8) for parameterdependent $G_{\mu \nu}$ and $B_{\mu \nu}$. In contrast, solutions to (9) with $K^{i} \neq 0$ have no counterparts among the solutions 
of (8). Such solutions may be called "dilatational" solutions, because in special cases they are true dilatations, as we will see in the example below (further examples can be found in $[12]$ ).

Finally we note that the solutions to equations (9) come in infinitely big families and that, as a consequence, the corresponding commutator algebra of the global symmetries is an infinite dimensional loop-like algebra. This has been observed already in 12 and is a consequence of the fact that the action depends on the $A_{m}^{i}$ only via their field strengths 15. All members of a family of solutions arise from one solution $V^{\mu}(X), K^{i}(y), p_{\mu}(X)$ by multiplying that solution with arbitrary functions of the $y^{i}$. As the $y^{i}$ are constant on-shell, this infinite dimensionality of the space of global symmetries has no practical importance, i.e., in order to discuss the global symmetries it is sufficient to consider just one representative of each family.

f. Example. To illustrate the results presented above, we specify them for a simple class of models characterized by Lagrangians containing only one $U(1)$ gauge field $A_{m}$ and the following choices for the background

$$
G_{y M}=B_{y \mu}=0, \quad G_{\mu \nu}=f(y) \eta_{\mu \nu}, \quad B_{\mu \nu}=B_{\mu \nu}(y),
$$

leading to

$$
\begin{aligned}
L / e= & -\frac{1}{2} h^{m n} \partial_{m} x^{\mu} \partial_{n} x^{\nu} G_{\mu \nu}+\frac{1}{2} \varepsilon^{m n} \partial_{m} x^{\mu} \partial_{n} x^{\nu} B_{\mu \nu} \\
& +\chi_{k} \gamma^{n} \gamma^{k} \psi^{\nu} \partial_{n} x^{\mu} G_{\mu \nu}-\frac{1}{4} \chi_{k} \gamma^{n} \gamma^{k} C \chi_{n} \bar{\psi}^{\mu} \psi^{\nu} G_{\mu \nu} \\
& +\frac{i}{2} \bar{\psi}^{\mu} \gamma^{m} \partial_{m} \psi^{\nu} G_{\mu \nu}-\frac{\mathrm{i}}{4} \bar{\psi}^{\nu} \gamma^{m} \gamma_{*} \psi^{\mu} \partial_{m} y \partial_{y} B_{\mu \nu} \\
& +\frac{1}{2} \varepsilon^{m n}\left(\partial_{m} A_{n}-\partial_{n} A_{m}\right) y
\end{aligned}
$$

where the auxiliary fields $F^{\mu}$ have been eliminated. As shown in 12], in this case the general solution of equations (7) is (modulo trivial global symmetries)

$$
\begin{aligned}
K & =2 r(y) \\
V^{\mu} & =-r(y)[\ln f(y)]^{\prime} x^{\mu}+r^{\mu}(y)+r^{[\mu \lambda]}(y) \eta_{\lambda \nu} x^{\nu} \\
a_{\mu} & =-V^{\lambda \prime} f(y) \eta_{\mu \lambda}, \quad a_{y}=0 \\
b_{\mu} & =\left(r(y) B_{\mu \nu}^{\prime}\right)^{\prime} x^{\nu}+B_{\mu \nu}^{\prime} V^{\nu}, \quad b_{y}=0 \\
p_{\mu} & =r(y) B_{\mu \nu}^{\prime} x^{\nu}+B_{\mu \nu} V^{\nu}, \quad p_{y}=0
\end{aligned}
$$

where a prime denotes differentiation with respect to $y$ and $r(y), r^{\mu}(y)$ and $r^{[\mu \lambda]}(y)$ are arbitrary functions of $y$ and correspond to families of dilatations, translations and Lorentz-transformations in target space, respectively. For three reasons the dilatations are special: (i) as discussed already above, they have no counterpart among the global symmetries of the ordinary superstring on a flat background; (ii) they can change the value of the "extra coordinate" $y$; (iii) they can map solutions to the classical equations of motion with vanishing field strength $\partial_{m} A_{n}-\partial_{n} A_{m}$ to solutions with non-vanishing field strength, in contrast to the translations and Lorentz-transformations. Property (ii) holds because of $\Delta y=2 r(y)$ and is intimately related to property (iii) because the field strength is related to $y$ by $f^{\prime}(y) \approx \varepsilon^{m n} \partial_{m} A_{n}+\ldots$ where $\approx$ is equality on-shell. We stress that the presence of the dilatations is not an artefact of our formulation. Rather, they are of course present even after elimination of $y$. Finally we note that properties (ii) and (iii) extend to more complicated backgrounds for which solutions to (9) with $K^{i} \neq 0$ exist.

g. Acknowledgements. FB thanks the ErwinSchrödinger-Institute for hospitality and financial support during the time when this work was completed, and was supported by a DFG habilitation grant at earlier stages of the work. AK was supported by ÖNB under project grant number 7731 and by "Fonds zur Förderung der Wissenschaftlichen Forschung" under project grant number P13125-TPH.

[1] P. Ramond, Phys. Rev. D 3 (1971) 2415; A. Neveu and J.H. Schwarz, Nucl. Phys. B 31 (1971) 86; Phys. Rev. D 4 (1971) 1109

[2] S. Deser and B. Zumino, Phys. Lett. B 65 (1976) 369; L. Brink, P. di Vecchia and P. Howe, Phys. Lett. B 65 (1976) 471

[3] E. Bergshoeff, S. Randjbar-Daemi, A. Salam, H. Sarmadi and E. Sezgin, Nucl. Phys. B 269 (1986) 77

[4] B. Brinne, S.E. Hjelmeland and U. Lindström, Phys. Lett. B 459 (1999) 507 (hep-th/9904175)

[5] E. Bergshoeff, L.A.J. London, P.K. Townsend, Class. Quantum Grav. 9 (1992) 2545 hep-th/9206026); P.K. Townsend, Phys. Lett. B 277 (1992) 285

[6] M. Cederwall, P.K. Townsend, Phys. Lett. B 409 (1997) 131 hep-th/9705160); JHEP 9709 (1997) 003 (hepth/9709002

[7] C.M. Hull, Nucl. Phys. B 468 (1996) 113 (hepth/9512181); C. Vafa, Nucl. Phys. B 469 (1996) 403 (hepth/9602022

[8] A. Kling, BRST cohomology of Dirichlet-Superstrings, diploma thesis, Vienna 1998 (unpublished)

[9] P.S. Howe, J. Phys. A: Math. Gen. 12 (1979) 393

[10] F. Brandt, W. Troost and A. Van Proeyen, Phys. Lett. B 374 (1996) 31 hep-th/9510195)

[11] F. Brandt, W. Troost and A. Van Proeyen, Nucl. Phys. B 464 (1996) 353 (hep-th/9509035)

[12] F. Brandt, J. Gomis and J. Simón, Phys. Lett. B 419 (1998) 148 (hep-th/9707063); Nucl. Phys. B 523 (1998) 623 (hep-th/9712125); Phys. Rev. Lett. 81 (1998) 1770 (hep-th/9803196)

[13] G. Barnich, F. Brandt and M. Henneaux, Commun. Math. Phys. 174 (1995) 57 (hep-th/9405109)

[14] B. de Wit and P. van Nieuwenhuizen, Nucl. Phys. B 312 (1989) 58; C.M. Hull and B. Spence, Phys. Lett. B 232 (1989) 204; Nucl. Phys. B 353 (1991) 379.

[15] F. Brandt, J. Gomis, D. Mateos and J. Simón, Phys. Lett. B 443 (1998) 147 (hep-th/9807113) 